\title{
Association of County-Level Racial Diversity and Likelihood of a Textbook Outcome Following Pancreas Surgery
}

\author{
Adrian Diaz, MD, MPH ${ }^{1,2,3}$, Djhenne Dalmacy, MS ${ }^{1}$, Chelsea Herbert, BS ${ }^{4}$, Rayyan S. Mirdad ${ }^{5}$, \\ J. Madison Hyer, MS ${ }^{1}$, and Timothy M. Pawlik, MD, MPH, PhD ${ }^{1}$ [1] \\ ${ }^{1}$ Department of Surgery, The Ohio State University Wexner Medical Center and James Comprehensive Cancer Center, \\ Columbus, $\mathrm{OH} ;{ }^{2}$ National Clinician Scholars Program at the Institute for Healthcare Policy and Innovation, University of \\ Michigan, Ann Arbor, MI; ${ }^{3}$ Center for Healthcare Outcomes and Policy, University of Michigan, Ann Arbor, MI; ${ }^{4}$ Ohio \\ University Heritage College of Osteopathic Medicine, Dublin, OH; ${ }^{5}$ Royal College of Surgeons, Dublin, Ireland
}

\begin{abstract}
Introduction. Residential racial desegregation has demonstrated improved economic and education outcomes. The degree of racial community segregation relative to surgical outcomes has not been examined.

Patients and Methods. Patients undergoing pancreatic resection between 2013 and 2017 were identified from Medicare Standard Analytic Files. A diversity index for each county was calculated from the American Community Survey. Multivariable mixed-effects logistic regression with a random effect for hospital was used to measure the association of the diversity index level with textbook outcome (TO).

Results. Among the 24,298 Medicare beneficiaries who underwent a pancreatic resection, most patients were male $(n=12,784,52.6 \%)$, White $(n=21,616,89 \%)$, and had a median age of 72 (68-77) years. The overall incidence of TO following pancreatic surgery was $43.3 \%$. On multivariable analysis, patients who resided in low-diversity areas had $16 \%$ lower odds of experiencing a TO following pancreatic resection compared with patients from high-diversity communities (OR $0.84,95 \%$ CI $0.72-0.98$ ).
\end{abstract}

Supplementary Information The online version contains supplementary material available at https://doi.org/10.1245/s10434021-10316-3.

(C) Society of Surgical Oncology 2021

First Received: 3 January 2021

Accepted: 1 May 2021;

Published Online: 18 June 2021

A. Diaz, MD, MPH

e-mail: Adriandi@med.umich.edu
Compared with patients who resided in the high-diversity areas, individuals who lived in low-diversity areas had higher odds of 90-day readmission (OR 1.16, 95\% CI 1.03-1.31) and had higher odds of dying within 90 days (OR 1.85, 95\% CI 1.45-2.38) (both $p<0.05$ ). Nonminority patients who resided in low-diversity areas also had a $14 \%$ decreased likelihood to achieve a TO after pancreatic resection compared with nonminority patients in high-diversity areas (OR $0.86,95 \%$ CI $0.73-1.00$ ).

Conclusion. Patients residing in the lowest racial/ethnic integrated counties were considerably less likely to have an optimal TO following pancreatic resection compared with patients who resided in the highest racially integrated counties.

The average life expectancy of individuals in the USA can vary by up to 30 years between neighboring counties. ${ }^{1}$ Specifically, higher income has been associated with greater longevity and life expectancy. ${ }^{2,3}$ Furthermore, lower socioeconomic status has also been linked to operative mortality, higher burden of chronic disease, and worse self-reported health status. ${ }^{4-6}$ Importantly, low socioeconomic status is more prevalent among the Black population, which may contribute to racial and ethnic health disparities observed across a myriad of health conditions, including surgical outcomes. $^{7-9}$ Because residential segregation is considered a key factor in perpetuating socioeconomic disparities, ${ }^{10}$ there is an emerging belief that residential segregation is also an underlying cause of health inequalities. ${ }^{11-13}$ 
Racial and ethnic disparities in access to surgical care as well as variations in postoperative outcomes have been previously described. ${ }^{7-9,14}$ Strategies to mitigate disparities, however, remain poorly understood. ${ }^{15}$ Desegregation has resulted in improved economic and educational outcomes for minority populations. ${ }^{16-18}$ According to Williams and Collins, residential segregation can affect health outcomes in several major ways. ${ }^{19}$ In particular, segregation can determine the quality of education and employment opportunities, contribute to the creation of pathogenic neighborhoods and housing conditions, constrain the practice of health behaviors and encourage unhealthy ones, and adversely affect access to high-quality health care. In contrast, more integrated communities may have greater economic and educational opportunities, which may lead to improved social determinates of health, increased access to health care, and greater overall health. In turn, desegregation of communities may help improve health-related outcomes, including surgical care. Racial and ethnic health disparities may, however, persist even within the most integrated communities because of other underlying factors. As such, evaluation of the extent of racial/ethnic diversity within a patient's area of residence relative to surgical outcomes may be important to identify disparities, as well as to define mitigation strategies.

The impact of residential segregation on health care outcomes has recently been highlighted by the coronavirus disease 2019 (COVID-19) epidemic, which has had a vastly greater impact on Black and Latino communities. ${ }^{20}$ While examined relative to COVID-19 and a small set of other medical conditions, ${ }^{12,13}$ the impact of residential segregation on postoperative surgical outcomes has largely not been investigated. Pancreatic resection is a complex surgical procedure with a high incidence of morbidity and mortality that is particularly sensitive to variations in care leading to disparate outcomes. ${ }^{21-23}$ As such, we designed a national cohort study to characterize postoperative outcomes after pancreatic surgery among patients in different counties with varying degrees of racial/ethnic diversity. In particular, we hypothesized that patients residing in areas of high diversity were more likely to have optimal "textbook outcomes" following pancreatic surgery compared with patients from less diverse areas.

\section{METHODS}

\section{Data Source}

Data from 2013 through 2017 were retrieved from the Medicare Inpatient and Outpatient Standard Analytic Files (SAFs), which are maintained by the Centers for Medicare and Medicaid Services (CMS). The SAFs include patient- level data on demographic characteristics, diagnoses, procedures, and expenditures. Patients who underwent a pancreatic resection were identified using the procedure codes (Appendix 1) of the ninth and tenth revision of the International Classification of Diseases (ICD-9-CM and ICD-10-CM). Primary diagnosis codes and the related frequencies are provided in Appendix 2. Because Medicare beneficiaries enrolled in HMOs have some or all their care paid for by payers other than Medicare, these claims are often not complete. As such, including beneficiaries enrolled in HMOs would introduce unknown missingness into the data and bias the results. Excluding Medicare beneficiaries enrolled in HMOs is well established and commonly utilized by investigators. If a patient underwent multiple procedures over the course of the study period, only the first procedure was included for the purposes of analysis. Approval was obtained from the institutional review board at the Ohio State University Wexner Medical Center.

\section{Variables}

The independent variable of interest was racial diversity at the county level. Using the county-level 2018 American Community Survey: 5-Year Data (2014-2018), obtained from the National Historical Geographic Information System (NHGIS), ${ }^{24}$ a diversity index (DI) was calculated for each county based on the Shannon' Diversity Index $(H)$ approach. ${ }^{25}$ The Shannon's diversity index $\left(H^{\prime}\right)$ is one of the most notable multigroup diversity measures that enable researchers to account for both richness (the number of species present) and evenness (the relative abundance of species). The index is obtained using the equation:

$$
H^{\prime}=-\sum_{j=1}^{s} P_{j} \ln \left(P_{j}\right)
$$

where $s$ represents the total number of species present, $P_{j}$ represents the proportion of individuals who belong to species $j$, and $H^{\prime}$ represent the level of uncertainty regarding the identity of a randomly selected individual. In other words, in a highly diverse community, predicting the species of any given individual should involve a high level of uncertainty; thus, we should have a relatively high $H^{\prime}$ compared with a less diverse community. ${ }^{26,27}$ To put in context, letting $s$ represent the number of races, $P_{j}$ the proportion of each race, we obtained $H^{\prime}$, the level of diversity in each county. Of note, the US Census Bureau proposed several measures of evenness to quantify racial segregation, one of which was developed by Theil. ${ }^{28}$ It has been demonstrated that the symmetric Theil index is related to the Shannon's diversity index. ${ }^{29}$ 
Population size information was obtained for nine different racial/ethnic group: "White alone," "Black or African American alone," "American Indian and Alaska Native alone," "Asian alone," "Native Hawaiian and Other Pacific Islander alone," "some other race alone," "two or more races," "two races including some other race," and "two races excluding some other race, and three or more races." For purposes of the current study, those that identified as two or more races/ethnicities were combined into one group. The diversity index values were standardized and categorized into three different levels: low segregation (below one standard deviation), average diversity (within one standard deviation), and high diversity (above one standard deviation). The dataset was then merged with the Medicare data for all subsequent analyses. Preoperative patient comorbidities were accounted for using the Charlson Comorbidity Index (CCI); additional independent variables included age, sex, minority status, hospital type (teaching versus nonteaching), hospital volume, expenditure, and surgery type (elective versus nonelective).

Textbook outcome (TO), a composite measure of surgical outcome based on several postoperative outcomes, was the main outcome of interest. ${ }^{22,30}$ A patient was categorized as having a TO if the patient did not experience any of the composite elements including no extended length of stay (LOS) ( $>75$ th percentiles LOS), did not have any complication, ${ }^{31}$ was not readmitted into any hospital within 90 days from discharge, and did not die within 90 days. ICD-9-CM and ICD-10-CM codes were used to identify postoperative complications including pulmonary failure, pneumonia, myocardial infarction, deep venous thrombosis, pulmonary embolism, renal failure, surgical site infection, gastrointestinal bleeding, and postoperative hemorrhage. These complications represent a subset of codes from administrative claims with the greatest sensitivity and specificity. ${ }^{31}$

\section{Statistical Analysis}

Descriptive statistics such as median (IQR) and proportions were calculated for continuous (nonnormal) and categorical variables, respectively. At the bivariate level, possible associations among patient demographics (e.g., age, minority status), clinical factors (e.g., CCI, elective surgery), and postoperative outcomes (e.g., TO and its components) as well as the diversity index (low, average, high) were assessed using Kruskal-Wallis test for interval (nonnormal) variables and $\chi^{2}$ tests for categorical variables. Multivariable mixed-effects logistic regression with a random effect for hospital was performed to measure the association of diversity index level with TO and its components while adjusting for confounding factors such as age, sex, minority status, hospital type (teaching versus nonteaching), hospital volume, expenditure, and surgery type (elective versus nonelective). Minority status was defined as any individual who did not identify as White (i.e., non-White). To assess a possible interaction effect of race and diversity, analyses using the mixed effects model were repeated to include a minority* diversity interaction as well as the main effects for diversity. To test for potential colinearity between race and the diversity index, a one-way analysis of variance and a multiple comparison test using the Bonferroni method were performed to assess pairwise differences for different race/ethnicity categories. These results were all statistically significant at alpha $=$ 0.05 , indicating that the mean of the diversity index was different across races. Additionally, when both race/ethnicity and the diversity index were included in the final model, there was model convergence. Finally, to test for variance inflation, the minority variable was removed from the final model for TO, and there was no evidence of variance inflation. These points have been added to the methods section. All statistical analyses were performed using SAS v 9.4 (SAS Institute, Cary, NC), and a statistical significance of $p=0.05$ was used.

\section{RESULTS}

Among the 24,298 Medicare beneficiaries who underwent a pancreatic resection, most patients were male $(n=$ $12,784,52.6 \%)$, were White $(n=21,616,89 \%)$, and had a median age of $72(68-77)$ years (Table 1$)$. The majority underwent an elective operation $(n=20,807,85.6 \%)$ at a teaching hospital ( $n=18,649,76.8 \%$ ). Overall, 16,864 (69.4\%) patients resided in a county with average diversity, while $3912(16.1 \%)$ patients resided in a county with low diversity and 4042 (16.2\%) with high diversity. Of note, there was a slightly larger proportion of male patients from low-diversity areas (low diversity: $n=1856$ (54.7\%) versus average diversity: $n=8855(52.5 \%)$ versus high diversity: $n=2073(51.3 \%) ; p=0.011)$. While there was a similar proportion of non-White patients (Black, $n=104,3.1 \%$; other race/ethnicity, $n=105,3.1 \%$ ) in low-diversity counties, there was a smaller proportion of non-White patients who resided in high-diversity counties (Black, $n=$ $180,4.5 \%$ versus other race/ethnicity, $n=374,9.3 \% ; p<$ $0.001)$. In addition, the overall proportion of patients who underwent elective surgery was lower in the low-diversity counties (low diversity: $n=2829(83.4 \%)$ versus average diversity: $n=14,395$ (85.4\%) versus high diversity: $n=$ $3583(88.6 \%) ; p<0.001)$.

The overall incidence of TO following pancreatic surgery was $43.3 \%$. Figure 1 shows the proportion of patients who achieved each component of a TO, as well as the 
TABLE 1 Bivariate analyses of patient characteristics versus level of diversity using the Shannon Diversity Index $(H)$

\begin{tabular}{|c|c|c|c|c|c|}
\hline Variable & Total $n=24,298$ & Low diversity $n=3392$ & Average diversity $n=16,864$ & High diversity $n=4042$ & $p$-value \\
\hline Age, median (IQR) & $72(68-77)$ & $73(69-77)$ & $72(68-77)$ & $72(68-77)$ & 0.09 \\
\hline Male & $12,784(52.6 \%)$ & $1856(54.7 \%)$ & $8855(52.5 \%)$ & $2073(51.3 \%)$ & 0.011 \\
\hline Race & & & & & $<0.001$ \\
\hline White & $21,616(89 \%)$ & $3183(93.8 \%)$ & $14,945(88.6 \%)$ & $3488(86.3 \%)$ & \\
\hline Black & $1503(6.2 \%)$ & $104(3.1 \%)$ & $1219(7.2 \%)$ & $180(4.5 \%)$ & \\
\hline Other & $1179(4.9 \%)$ & $105(3.1 \%)$ & $700(4.2 \%)$ & $374(9.3 \%)$ & \\
\hline Elective surgery & $20,807(85.6 \%)$ & $2829(83.4 \%)$ & $14,395(85.4 \%)$ & $3583(88.6 \%)$ & $<0.001$ \\
\hline Teaching hospital & $18,649(76.8 \%)$ & $2549(75.1 \%)$ & $12,921(76.6 \%)$ & $3179(78.6 \%)$ & 0.001 \\
\hline LOS, median (IQR) & $8(6-14)$ & $9(6-15)$ & $8(6-14)$ & $8(6-13)$ & $<0.001$ \\
\hline CCS, median (IQR) & $2(2-8)$ & $3(2-8)$ & $2(2-8)$ & $3(2-8)$ & $<0.001$ \\
\hline Expenditure index (kUSD) & $22.5(16.9-37.0)$ & $21.8(16.8-36.6)$ & $23.4(17.0-37.4)$ & $21.0(16.7-35.1)$ & $<0.001$ \\
\hline
\end{tabular}

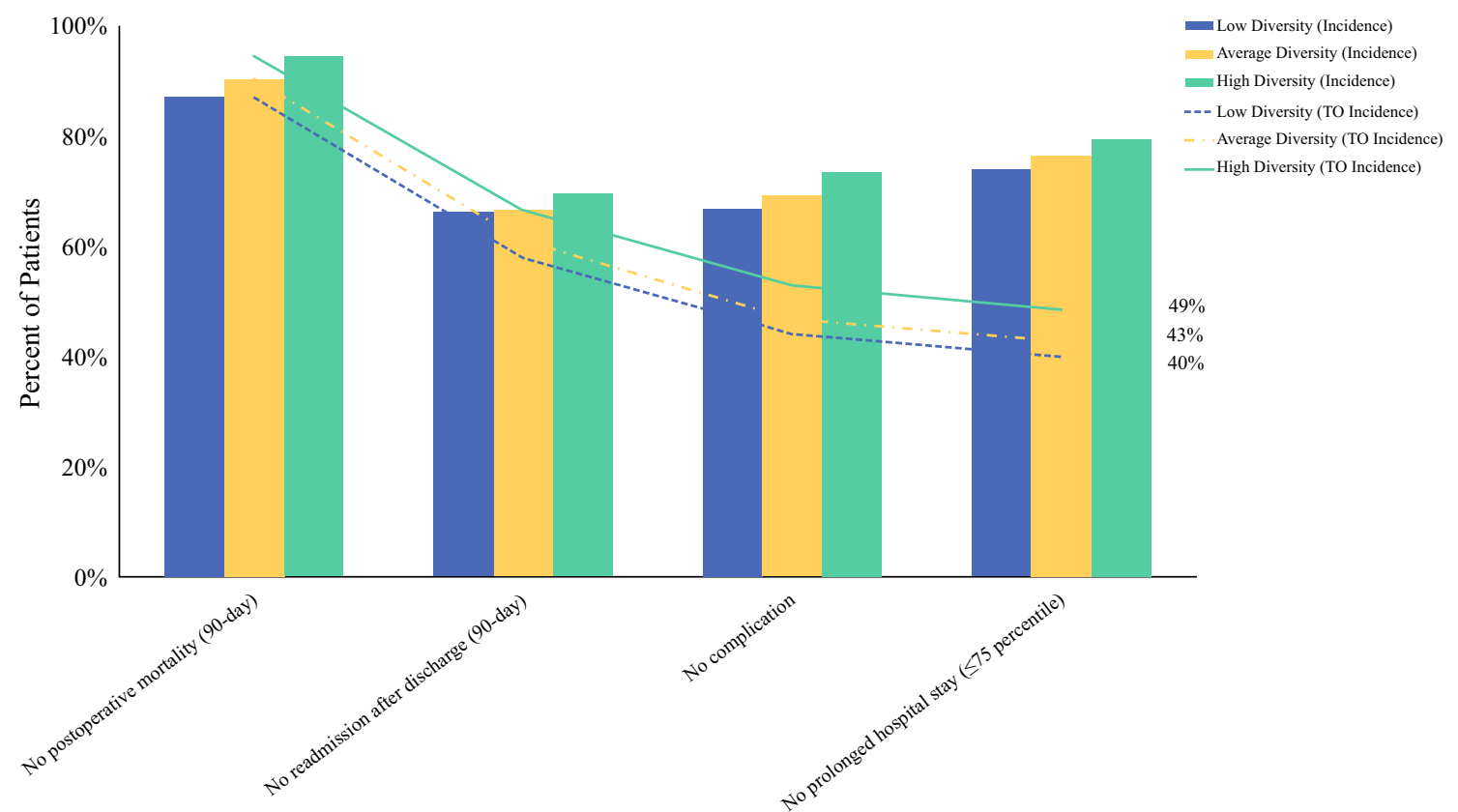

FIG. 1 Proportion of patients achieving TO and individual components of TO stratified by Diversity. TO textbook outcome

proportion of patients who achieved a TO stratified by diversity index (Fig. 1). While not experiencing a complication had the greatest impact on the ability to obtain a TO (no complications, $n=16,902,30.4 \%$ ), no mortality within 90 days ( $n=22,034,90.7 \%$ ) was the outcome most commonly achieved among patients. In addition, $76.8 \%$ ( $n=18,651$ ) of the patients did not have an extended LOS, and $67.0 \%(n=16,287)$ did not experience a hospital readmission within 90 days of discharge. The unadjusted likelihood of achieving a TO was lower among patients in low-diversity areas (low diversity: $n=1354$ (39.9\%) versus average diversity: $n=7204(42.7 \%)$ versus high diversity: $n=1964$ (48.6\%); $p<0.001$ ) (Table 2). In particular, several TO components varied markedly according to county diversity status. For example, patients who resided in low-diversity areas had a longer median LOS (low diversity: 9 days (IQR 6, 15) versus average diversity: 8 days (IQR 6, 14) versus high diversity: 8 days (IQR 6, 13); $p<0.001$ ), and had a higher likelihood of 90-day mortality (low diversity: $n=427$ (12.6\%) versus average diversity: $n=1618$ (9.6\%) versus high diversity: $n=219$ (5.4\%); $p<$ $0.001)$. In contrast, patients who resided in high-diversity areas had better outcomes across the range of individual TO component metrics including risk of complications, 90-day readmission, and death within 90 days following pancreatic surgery (all $p<0.05$ ) (Table 2). 
TABLE 2 Bivariate analyses of postoperative outcomes versus level of diversity using the Shannon Diversity Index $(H)$

\begin{tabular}{|c|c|c|c|c|c|}
\hline Outcomes & Total $n=24,298$ & Low diversity $n=3392$ & Average diversity $n=16,864$ & High diversity $n=4042$ & $p$-value \\
\hline Textbook outcome & $10,522(43.3 \%)$ & $1354(39.9 \%)$ & $7204(42.7 \%)$ & $1964(48.6 \%)$ & $<0.001$ \\
\hline Extended LOS & $5647(23.2 \%)$ & $878(25.9 \%)$ & $3945(23.4 \%)$ & $824(20.4 \%)$ & $<0.001$ \\
\hline Complication & $7396(30.4 \%)$ & $1128(33.3 \%)$ & $5201(30.8 \%)$ & $1067(26.4 \%)$ & $<0.001$ \\
\hline \multicolumn{6}{|l|}{ Readmission } \\
\hline 30 days & $5478(22.5 \%)$ & $774(22.8 \%)$ & $3852(22.8 \%)$ & $852(21.1 \%)$ & 0.051 \\
\hline 90 days & $8011(33 \%)$ & $1148(33.8 \%)$ & $5637(33.4 \%)$ & $1226(30.3 \%)$ & $<0.001$ \\
\hline \multicolumn{6}{|l|}{ Mortality } \\
\hline 30 days & $1455(6 \%)$ & $290(8.5 \%)$ & $1023(6.1 \%)$ & $142(3.5 \%)$ & $<0.001$ \\
\hline 90 days & $2264(9.3 \%)$ & $427(12.6 \%)$ & $1618(9.6 \%)$ & $219(5.4 \%)$ & $<0.001$ \\
\hline
\end{tabular}

After adjustment for other competing risk factors on multivariable analyses including age, sex, non-White status, preoperative comorbidities, hospital type, and volume, the diversity index remained strongly associated with TO. Specifically, patients who resided in low-diversity areas had $16 \%$ lower odds to experience a TO following pancreatic resection compared with patients from highdiversity communities (OR 0.84, 95\% CI 0.72-0. 98) (Table 3). The effect of community diversity on likelihood to achieve TO persisted among patients from average versus high-diversity areas, albeit with the impact of diversity being slightly less than for low-diversity areas (OR 0.87, 95\% CI 0.77-0.99). Readmission within 90 days as well as mortality within 90 days were strongly associated with diversity. Specifically, compared with patients who resided in high-diversity areas, individuals who lived in low-diversity areas had higher odds of 90-day readmission (OR 1.16, 95\% CI 1.03-1.31) and higher odds of dying within 90 days (OR 1.85, 95\% CI 1.45-2.38) (both $p<0.05)$. While patients from an average-diversity community also had an increased risk of 90-day readmission (OR 1.15, 95\% CI 1.04-1.26) and 90-day mortality (OR

TABLE 3 Multivariable logistic regression assessing the effect of level of diversity on postoperative outcomes (against reference high diversity)

\begin{tabular}{llllll}
\hline Outcomes & \multicolumn{2}{l}{ Low diversity } & & \multicolumn{2}{c}{ Average diversity } \\
\cline { 2 - 3 } & OR & $95 \%$ CI & & OR & $95 \%$ CI \\
\hline Textbook outcome & $\mathbf{0 . 8 4}$ & $\mathbf{0 . 7 2 - 0 . 9 8}$ & & $\mathbf{0 . 8 7}$ & $\mathbf{0 . 7 7 - 0 . 9 9}$ \\
Extended LOS & 0.91 & $0.74-1.12$ & & 0.95 & $0.80-1.14$ \\
Complications at index & 1.15 & $0.97-1.37$ & & 1.13 & $0.97-1.31$ \\
90-day readmission & $\mathbf{1 . 1 6}$ & $\mathbf{1 . 0 3 - 1 . 3 1}$ & & $\mathbf{1 . 1 5}$ & $\mathbf{1 . 0 4 - 1 . 2 6}$ \\
90-day mortality & $\mathbf{1 . 8 5}$ & $\mathbf{1 . 4 5 - 2 . 3 8}$ & & $\mathbf{1 . 5 5}$ & $\mathbf{1 . 2 5}-\mathbf{1 . 9 2}$ \\
\hline
\end{tabular}

Bold values are statistically significant $(p<0.05)$
1.55 , 95\% CI 1.25-1.92) compared with patients from high-diversity areas, the size of the effect was not as pronounced as among patients from low-diversity areas.

Of note, additional stratified analyses to examine the interaction of race/ethnicity and community diversity demonstrated an increased probability to achieve a TO as the diversity index increased among both White and nonWhite patients (Fig. 2). Specifically, in assessing patients stratified by minority status, non-White patients who lived in low-diversity areas had lower odds of achieving a postoperative TO following pancreatic resection compared with non-White patients who resided in high-diversity areas (OR 0.69, 95\% CI 0.48-0.99) (Table 4). The lower probability to achieve a TO was largely attributable to higher odds of 90-day readmission (OR 1.51, 95\% CI 1.08-2.13) and 90-day mortality (OR 2.97, 95\% CI 1.70-5.17). A similar, albeit less pronounced, association of neighborhood diversity with postoperative outcomes was noted among White patients. Specifically, White patients who resided in low-diversity areas had a $14 \%$

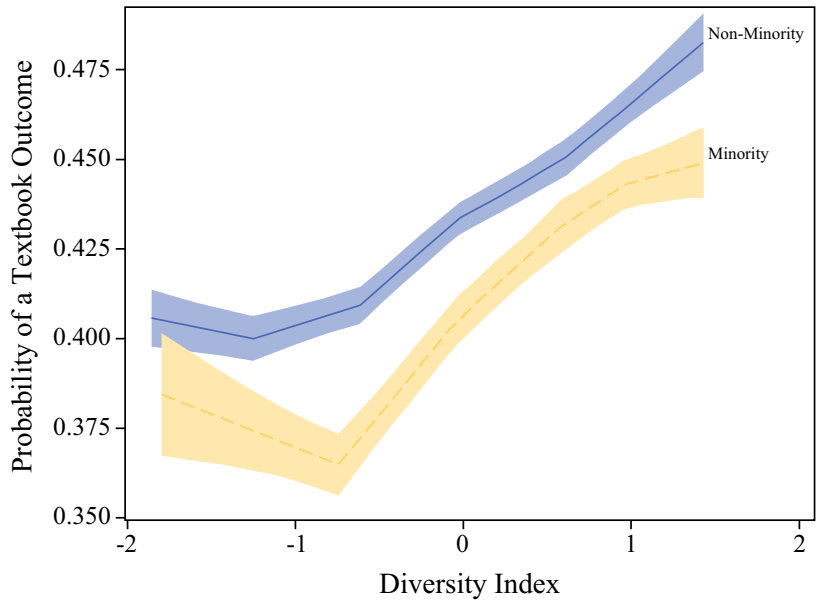

FIG. 2 Plot of probability of achieving a textbook outcome based on diversity stratified by minority status 
TABLE 4 Multivariable logistic regression assessing the effect of level of diversity on postoperative outcomes stratified by minority status (against reference high diversity)

\begin{tabular}{|c|c|c|c|c|}
\hline \multirow[t]{2}{*}{ Outcome } & \multicolumn{2}{|c|}{ Low diversity } & \multicolumn{2}{|c|}{ Average diversity } \\
\hline & OR & $95 \% \mathrm{CI}$ & OR & $95 \% \mathrm{CI}$ \\
\hline \multicolumn{5}{|l|}{ Minority } \\
\hline Textbook outcome & 0.69 & $0.48-0.99$ & 0.84 & $0.67-1.04$ \\
\hline Extended LOS & 1.13 & $0.75-1.69$ & 1.03 & $0.78-1.35$ \\
\hline Complications at index & 1.42 & $0.98-2.05$ & 1.28 & $1.00-1.63$ \\
\hline 90-day readmission & 1.51 & $1.08-2.13$ & 1.15 & $0.93-1.43$ \\
\hline 90-day mortality & 2.97 & $1.70-5.17$ & 1.78 & 1.16-2.74 \\
\hline \multicolumn{5}{|l|}{ Nonminority } \\
\hline Textbook outcome & 0.86 & $0.73-1.00$ & 0.88 & $0.77-1.00$ \\
\hline Extended LOS & 0.88 & $0.71-1.09$ & 0.94 & $0.78-1.12$ \\
\hline Complications at index & 1.12 & $0.93-1.34$ & 1.10 & $0.94-1.28$ \\
\hline 90-day readmission & 1.14 & $1.00-1.29$ & 1.14 & 1.03-1.27 \\
\hline 90-day mortality & 1.76 & $1.35-2.28$ & 1.51 & $1.20-1.90$ \\
\hline
\end{tabular}

Bold values are statistically significant $(p<0.05)$

decreased likelihood to achieve a TO after pancreatic resection compared with White patients in high-diversity areas (OR 0.86, 95\% CI 0.73-1.00). In particular, White patients in low-diversity areas had higher odds of 90-day mortality compared with individuals who lived in highdiversity areas (OR 1.76, 95\% CI 1.35-2.28).

\section{DISCUSSION}

Societal racial/ethnic disparities are important determinants of health that can drive variation in health care outcomes. ${ }^{7,9,12,32,33}$ The fact that some populations experience more social determinants of health than others can result in health disparities and health inequity.

Whereas health disparities are the patterns one observes related to health among different patient populations, health equity, or health inequity, is related more to the causes of health disparity. In essence, health inequity relates to the structural or institutional patterns that ultimately result in health disparities. ${ }^{34}$ One such structural mechanism may be racial/ethnic residential segregation. ${ }^{10,11}$ To this point, Arcaya and Schnake-Mahl have argued that residential segregation independent of race and/ or income has an adverse impact on health. ${ }^{35}$ While this topic has garnered considerable interest relative to the novel coronavirus epidemic, the impact of residential segregation on health care outcomes among a surgical population has not been previously examined. The current study was therefore important because we specifically examined surgical outcomes after pancreatic resection relative to county-level diversity. Pancreatic resection was used as the index procedure given its relatively high morbidity and mortality, as well as data suggesting variation in outcomes relative to access to care. ${ }^{36-38}$ In addition, we used the diversity index, which was a validated tool utilized by the Census Bureau to measure residential segregation. $^{28}$ Of note, patients from the lowest racially integrated counties had $16 \%$ lower odds to experience a TO following pancreatic resection compared with patients from high-diversity communities. Differences in the odds to achieve a TO were largely attributable to differences in the odds of perioperative death, as well as readmission, among patients in low-integrated neighborhoods. Interestingly, the effect of diversity was incremental as patients from counties with an average diversity index had lower odds of an optimal outcome following pancreatic resection compared with individuals from high-diversity areas, yet not as pronounced as patients from low-diversity neighborhoods. In addition, while the impact of poor diversity was more pronounced among non-White patients, both non-White and White patients in the lowest racially integrated counties had worse outcomes, including higher odds of mortality.

Racial/ethnic segregation has a long and troubled history in the USA that has perpetuated systemic disparities and unequal community investment. ${ }^{39,40}$ For example, Blacks and Hispanics who live in highly segregated and isolated neighborhoods have lower housing quality, higher concentrations of poverty, and less access to good jobs and education. Disciplines outside of health care have demonstrated how lack of racial diversity can be associated with worse economic and educational attainment, ${ }^{17,18}$ both of which are considered important factors of social determinants of health. Social determinants of health-the conditions in the environments in which people are born, live, learn, work, play, worship, and age — can affect a wide range of health, functioning, and quality-of-life outcomes and risks. ${ }^{41}$ Patients living in areas heavily influenced by social determinants of health may experience greater stress and have a higher risk of illness and death. ${ }^{42}$ To this point, our group and others have demonstrated an association between social determinants of health in a particular geographic area and a myriad of health outcomes. ${ }^{14,43-46}$ In particular, Azap et al. recently reported that social vulnerability was associated with adverse postoperative surgical outcomes after hepatopancreatic surgery. ${ }^{44}$ The current study built off this prior work to demonstrate that county-level racial diversity itself was associated with postoperative outcomes following complex surgical procedures such as pancreatectomy. Specifically, patients who resided in communities with low diversity had roughly a 1 in 6 lower chance to achieve an optimal TO following pancreatic resection. 
The lower incidence of TO among patients from lowdiversity counties was largely attributable to a higher incidence of readmission and 90-day mortality in those communities. Faiz et al. had noted that variation in readmission after complex surgery was associated with patientlevel factors, including race/ethnicity. ${ }^{47}$ In addition, risk of readmission has been strongly linked to resources available at the time of discharge including home health services, rehabilitation, and personal/familial finances/support. ${ }^{48-50}$ Pancreatic resection is also a surgical procedure that is highly sensitive to the volume-outcome relationship, as well as the overall quality of the hospital in which the procedure is performed. ${ }^{21,38,51}$ As such, disparities in 90-day mortality following pancreatectomy may be attributed to differences in access to high-quality hospitals. For example, Sarrazin et al. reported that Medicare beneficiaries who were from highly segregated areas were more likely to be hospitalized for acute myocardial infarction in a high-mortality hospital. ${ }^{52}$ In a separate study, Dimick et al. reported that Black patients were 58\% more likely than White patients to undergo high-risk surgical procedures in lower-quality hospitals despite living in close proximity to high-quality centers. The chance of having surgery at a low-quality hospital further increased among Black individuals who lived in segregated communities. ${ }^{53}$ In the current study, after controlling for several factors including hospital teaching status and hospital volume, the diversity index remained associated with worse postpancreatectomy outcomes and a lower incidence of TO. In turn, the data collectively suggest that achieving better outcomes for patients will require a focus not only on hospital-specific factors, but also community-level social determinants of health such as residential diversity.

Several limitations should be considered when interpreting the results of the current study. As with other studies using administrative data, the findings were subject to residual confounding due to unmeasured factors such as noncoded comorbidities. Additionally, the administrative claims database utilized did not have patient-level information on socioeconomic status. The diversity index was calculated at only one point in time (i.e., cross sectional analysis); therefore, the study was unable to assess longitudinal changes in county-level diversity and any potential effects on postoperative outcomes. The analytic cohort was also limited to patients over the age of 65 years. In turn, the findings may not be generalizable to younger patients. Despite these limitations, the observed differences in TO between high- versus low-diversity counties were notable and should compel further research to mitigate racial/ethnic disparities.

In conclusion, patients residing in low racial/ethnic integrated counties were considerably less likely to have an optimal TO following pancreatic resection compared with patients who resided in the more integrated counties. Importantly, differences in mortality were observed across levels of racial diversity for minority and nonminority patients. These findings highlight the important and farreaching implications of increasing residential racial/ethnic diversity and diversity. The data also serve to emphasize the need for health care leaders to invest in addressing social determinants of health through equitable community investment. ${ }^{54-57}$ Future research should seek to investigate the role of specific demographic information such as socioeconomic status (SES) and educational attainment on the association between diversity index and postoperative outcomes. In addition, future studies could consider how longitudinal changes to racial diversity may affect patientspecific risk factors to ensure access to surgical care, as well as equitable outcomes among all patients.

FUNDING Dr. Diaz receives funding from the University of Michigan Institute for Healthcare Policy and Innovation Clinician Scholars Program and salary support from the Veterans Affairs Office of Academic Affiliations during the time of this study.

\section{DISCLOSURES None.}

\section{REFERENCES}

1. Graham GN. Why your ZIP code matters more than your genetic code: promoting healthy outcomes from mother to child. Breastfeed Med Off J Acad Breastfeed Med. 2016;11:396-7.

2. Chetty R, Stepner M, Abraham S, et al. The association between income and life expectancy in the United States, 2001-2014. JAMA. 2016;315:1750.

3. Braveman PA, Cubbin C, Egerter S, et al. Socioeconomic disparities in health in the United States: what the patterns tell us. Am J Public Health. 2010;100(Suppl 1):S186-196.

4. Bennett KM, Scarborough JE, Pappas TN, et al. Patient socioeconomic status is an independent predictor of operative mortality. Ann Surg. 2010;252:552-7; discussion 557-8.

5. Puckrein GA, Egan BM, Howard G. Social and medical determinants of cardiometabolic health: the big picture. Ethn Dis. 2015;25:521-4.

6. Meyer OL, Castro-Schilo L, Aguilar-Gaxiola S. Determinants of mental health and self-rated health: a model of socioeconomic status, neighborhood safety, and physical activity. Am J Public Health. 2014;104:1734-41.

7. Mehtsun WT, Figueroa JF, Zheng J, et al. Racial disparities in surgical mortality: the gap appears to have narrowed. Health Aff Proj Hope. 2017;36:1057-64.

8. Rangrass G, Ghaferi AA, Dimick JB. Explaining racial disparities in outcomes after cardiac surgery: the role of hospital quality. JAMA Surg. 2014;149:223-7.

9. Nathan H, Frederick W, Choti MA, et al. Racial disparity in surgical mortality after major hepatectomy. J Am Coll Surg. 2008;207:312-9.

10. Massey DS. American Apartheid: segregation and the making of the underclass. Am J Sociol. 1990;96:329-57.

11. Popescu I, Duffy E, Mendelsohn J, et al. Racial residential segregation, socioeconomic disparities, and the White-Black survival gap. PloS ONE. 2018;13:e0193222. 
12. Kershaw KN, Pender AE. Racial/ethnic residential segregation, obesity, and diabetes mellitus. Curr Diab Rep. 2016;16:108.

13. Landrine H, Corral I, Lee JGL, et al. Residential segregation and racial cancer disparities: a systematic review. J Racial Ethn Health Disparities. 2017;4:1195-205.

14. Diaz A, Chavarin D, Paredes AZ, Tsilimigras DI, Pawlik TM. Association of neighborhood characteristics with utilization of high-volume hospitals among patients undergoing high-risk cancer surgery. Ann Surg Oncol. 2021;28(2):617-31. https://doi. org/10.1245/s10434-020-08860-5

15. Thornton RLJ, Glover CM, Cené CW, et al. Evaluating strategies for reducing health disparities by addressing the social determinants of health. Health Aff (Millwood). 2016;35:1416-23.

16. Enos RD. The space between us: social geography and politics. Cambridge University Press; 2017.

17. Johnson RC. Long-run impacts of school desegregation \& school quality on adult attainments. Working paper 16664; National Bureau of Economic Research. Epub ahead of print January 2011. https://doi.org/10.3386/w16664.

18. School segregation undermines U.S. economic mobility and dynamism. Equitable growth. 2019. http://www.equitablegrowth. org/school-segregation-undermines-u-s-economic-mobility-and-d ynamism/. Accessed 13 Oct 2020.

19. Williams DR, Collins C. Racial residential segregation: a fundamental cause of racial disparities in health. Public Health Rep. 2001;116:404-16.

20. Residential segregation plays a role in coronavirus disparities, study finds - the Washington post available from: https://www. washingtonpost.com/. Accessed 19 Oct 2020.

21. Amini N, Spolverato G, Kim Y, et al. Trends in hospital volume and failure to rescue for pancreatic surgery. J Gastrointest Surg Off J Soc Surg Aliment Tract. 2015;19:1581-92.

22. Merath K, Chen Q, Bagante F, et al. Textbook outcomes among Medicare patients undergoing hepatopancreatic surgery. Ann Surg. 2020;271:1116-23.

23. Birkmeyer JD, Stukel TA, Siewers AE, et al. Surgeon volume and operative mortality in the United States. $N$ Engl J Med. 2003;349:2117-27.

24. Manson S, Schroeder J, Van Riper D, et al. IPUMS national historical geographic information system: version 15.0. https://d oi.org/10.18128/D050.V15.0. Accessed 13 Oct 2020.

25. Shannon CE. A mathematical theory of communication. Bell Syst Tech J. 1948;27:379-423.

26. Morris EK, Caruso T, Buscot F, et al. Choosing and using diversity indices: insights for ecological applications from the German Biodiversity Exploratories. Ecol Evol. 2014;4:3514-24.

27. Ifo SA, Moutsambote J-M, Koubouana F, et al. Tree species diversity, richness, and similarity in intact and degraded forest in the tropical rainforest of the Congo Basin: case of the Forest of Likouala in the Republic of Congo. Int $J$ For Res. 2016;2016:7593681.

28. Bureau UC. Appendix B: measures of residential segregation. The United States census bureau. https://www.census.gov/topic s/housing/housing-patterns/guidance/appendix-b.html. Accessed 15 Oct 2020.

29. Ausloos M, Miśkiewicz J. Introducing the q-Theil index. Braz $J$ Phys. 2009;39:388-95.

30. Merath K, Chen Q, Bagante F, et al. A multi-institutional international analysis of textbook outcomes among patients undergoing curative-intent resection of intrahepatic cholangiocarcinoma. JAMA Surg. 2019;154:e190571.

31. Iezzoni LI, Daley J, Heeren T, et al. Identifying complications of care using administrative data. Med Care. 1994;32:700-15.

32. Wood MH, Carlin AM, Ghaferi AA, et al. Association of race with bariatric surgery outcomes. JAMA Surg. 2019;154:e190029e190029.
33. Zogg CK, Scott JW, Jiang W, et al. Differential access to care: the role of age, insurance, and income on race/ethnicity-related disparities in adult perforated appendix admission rates. Surgery. 2016;160:1145-54.

34. PatientEngagementHIT. What is the difference between health disparities, equity? PatientEngagementHIT. 2020. https://patiente ngagementhit.com/news/what-is-the-difference-between-health-d isparities-equity. Accessed 19 Oct 2020.

35. Arcaya M, Tucker-Seeley R, Kim R, et al. Research on neighborhood effects on health in the United States: a systematic review of study characteristics. Soc Sci Med. 1982;2016(168):16-29.

36. Diaz A, Burns S, Paredes AZ, et al. Accessing surgical care for pancreaticoduodenectomy: patient variation in travel distance and choice to bypass hospitals to reach higher volume centers. J Surg Oncol. 2019;120:1318-26.

37. Diaz A, Pawlik TM. Optimal location for centralization of hospitals performing pancreas resection in California. JAMA Surg. 2020;155(3):261-3. https://doi.org/10.1001/jamasurg.2019.4937

38. Nathan H, Cameron JL, Choti MA, et al. The volume-outcomes effect in hepato-pancreato-biliary surgery: hospital versus surgeon contributions and specificity of the relationship. J Am Coll Surg. 2009;208:528-38.

39. Rothstein R. The color of law: a forgotten history of how our government segregated America. New York, London: Liveright Publishing Corporation, A Division of W.W. Norton \& Company; 2017.

40. Racial wealth inequality: social problems and solutions. https:// www.brookings.edu/events/racial-wealth-inequality-social-probl ems-and-solutions/. Accessed 26 Sept 2020.

41. Cole BL, Fielding JE. Health impact assessment: a tool to help policy makers understand health beyond health care. Annu Rev Public Health. 2007;28:393-412.

42. What's the connection between residential segregation and health? RWJF. 2018. http://www.rwjf.org/en/culture-of-health/2 016/03/what_s_the_connectio.html. Accessed 19 Oct 2020.

43. Diaz A, Barmash E, Azap R, Paredes AZ, Hyer JM, Pawlik TM. Association of county-level social vulnerability with elective versus non-elective colorectal surgery. J Gastrointest Surg. 2021;25(3):786-94. https://doi.org/10.1007/s11605-020-04768-3

44. Azap RA, Paredes AZ, Diaz A, et al. The association of neighborhood social vulnerability with surgical textbook outcomes among patients undergoing hepatopancreatic surgery. Surgery. 2020;168:868-75.

45. Hu J, Kind AJH, Nerenz D. Area deprivation index predicts readmission risk at an urban teaching hospital. Am J Med Qual. 2018;33:493-501.

46. Kurani SS, McCoy RG, Lampman MA, et al. Association of neighborhood measures of social determinants of health with breast, cervical, and colorectal cancer screening rates in the US Midwest. JAMA Netw Open. 2020;3:e200618.

47. Gani F, Lucas DJ, Kim Y, et al. Understanding variation in 30-day surgical readmission in the era of accountable care: effect of the patient, surgeon, and surgical subspecialties. JAMA Surg. 2015;150:1042-9.

48. Dotson JL, Kappelman MD, Chisolm DJ, et al. Racial disparities in readmission, complications, and procedures in children with Crohn's disease. Inflamm Bowel Dis. 2015;21:801-8.

49. Using social determinants of health to predict readmissions. Healthcare innovation. 2019. https://www.hcinnovationgroup.co $\mathrm{m} /$ population-health-management/social-determinants-of-health/ article/21105919/using-social-determinants-of-health-to-predict-r eadmissions. Accessed 19 Oct 2020.

50. Chen Q, Merath K, Olsen G, et al. Impact of post-discharge disposition on risk and causes of readmission following liver and 
pancreas surgery. J Gastrointest Surg Off J Soc Surg Aliment Tract. 2018;22:1221-9.

51. Mehta R, Paredes AZ, Tsilimigras DI, Moro A, Sahara K, Farooq A, Dillhoff M, Cloyd JM, Tsung A, Ejaz A, Pawlik TM. Influence of hospital teaching status on the chance to achieve a textbook outcome after hepatopancreatic surgery for cancer among Medicare beneficiaries. Surgery. 2020;168(1):92-100. https://doi. org/10.1016/j.surg.2020.02.024

52. Sarrazin MV, Campbell M, Rosenthal GE. Racial differences in hospital use after acute myocardial infarction: does residential segregation play a role? Health Aff Proj Hope. 2009;28:w368378.

53. Dimick J, Ruhter J, Sarrazin MV, et al. Black patients more likely than whites to undergo surgery at low-quality hospitals in segregated regions. Health Aff Proj Hope. 2013;32:1046-53.

54. Thomas-Henkel C, Schulman M. Screening for social determinants of health in populations with complex needs: implementation considerations. Center for Health Care Stradegies Inc; 2020.

55. Bamdad MC, Englesbe MJ. Surgery and population health-redesigning surgical quality for greater impact. JAMA Surg. 2020. h ttps://doi.org/10.1001/jamasurg.2020.0808

56. H.R.6637-116th Congress (2019-2020): health equity and accountability act of 2020. 2020. https://www.congress.gov/bill/ 116th-congress/house-bill/6637. Accessed 9 Oct 2020.

57. Alley DE, Asomugha CN, Conway PH, et al. Accountable health communities-addressing social needs through Medicare and Medicaid. N Engl J Med. 2016;374:8-11.

Publisher's Note Springer Nature remains neutral with regard to jurisdictional claims in published maps and institutional affiliations. 